\title{
ESTADO DEL ARTE EN SOFTWARE EDUCATIVO
}

\section{STATE OF THE ART IN EDUCATIONAL SOFTWARE}

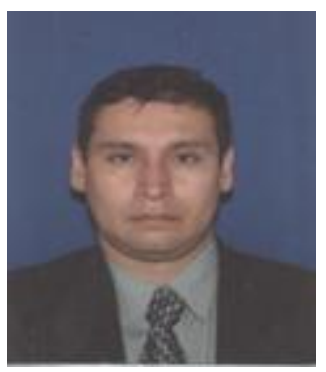

Mario Dustano Contreras Castro ${ }^{1}$

Este artículo evidencia la exploración, profundización, seguimientos, retroalimentación, aportes de las experiencias como aprendizajes de software educativo.

\section{RESUMEN}

Se contextualiza inicialmente en Diseño Instruccional en cuanto a su definición, modelos instruccionales, desarrollo en el Modelo ADDIE (Análisis, Diseño, Desarrollo, Implementación, Ejecución), como también, MEC (Modelo Educativo Computarizado).

Luego, se colocan los referentes de Edumatica como experiencia, aportes y renovación de Metodología de Software Educativo orientados a objetos de Conocimiento de Formación y Estudio como su representación de Escenas, Esquema, Navegación.

PALABRAS CLAVES: ADDIE, MEC, Edumatica, Objeto de Conocimiento, Objeto de Formación, Objeto de Estudio, Escenas, Esquema, Navegación.

\section{SUMMARY}

It was initially contextualized in Instructional Design as to its definition, instructional models, development in the ADDIE Model (Analysis, Design, Development, Implementation, Execution) as well, MEC (Educational Model Computerized). Then, the referents of Edumatica as experience, contributions and renewal Methodology of Educational Software Object Oriented Knowledge Training and study their representation Scene, Scheme, Navigation are placed.

KEYWORDS: ADDIE, MEC, Edumatica Object Knowledge Object Training, Object Study, Scenes, Scheme, Navigation.

\footnotetext{
${ }^{1}$ Ingeniero de Sistemas. Especialista en Multimedia Educativa. Cursando Maestría en Edumatica Fundación Universidad Autónoma de Colombia. Docente de Tiempo Completo, Programa Ingeniería en Informática, Facultad de Ciencia y Tecnologías, VUAD, Universidad Santo Tomas Sede Bogotá, mariocontreras@ustadistancia.edu.co, Docente Cátedra Tiempo Indefinido, Fundación Universidad Autónoma de Colombia, mario.contreras@fuac.edu.co
} 


\section{PRIMER ANTECEDENTE. DISEÑO INSTRUCCIONAL Que es Diseño Instruccional}

Bruner (1969) concibe el propósito de la instrucción como procurar los medios y los diálogos necesarios para traducir la experiencia en sistemas más eficaces de notación y ordenación. Esto Bruner lo considera como el centro de la educación, y sintetiza la idea diciendo que el diseño instruccional se ocupa de la planeación, la preparación y el diseño de los recursos y ambientes necesarios para que se lleve a cabo el aprendizaje.

Reigeluth (1983) define al diseño instruccional como la disciplina interesada en prescribir métodos óptimos de instrucción, al crear cambios deseados en los conocimientos y habilidades del estudiante.

Berger y Kam (1996) determina que el diseño instruccional como proceso es el desarrollo sistemático de los elementos instruccionales, usando las teorías del aprendizaje y las teorías instruccionales para asegurar la calidad de la instrucción. Incluye el análisis de necesidades de aprendizaje, los objetivos o competencias, el desarrollo de tareas y materiales, la evaluación del aprendizaje y el seguimiento del curso.

Broderick (2001) define el diseño instruccional como el arte y ciencia aplicada de crear un ambiente instruccional y los materiales, claros y efectivos, que ayudarán al alumno a desarrollar la capacidad para lograr ciertas tareas.

Richey, Fields y Foson (2001) especifican que Diseño Instruccional supone una planificación instruccional sistemática que incluye la valoración de necesidades, el desarrollo, la evaluación, la implementación y el mantenimiento de materiales y programas.

Hortom (Horton y otros, 2000) determina que el diseño instruccional para la educación a distancia y virtual se elabora siguiendo cualquier modelo, siempre y cuando se aborden sus elementos y se mantenga una relación con los principios pedagógicos de aprendizaje convirtiéndose este en la arquitectura del aprendizaje. En la clase presencial, la mayor parte del diseño instruccional de un curso está implícito en la experiencia y la sabiduría del facilitador, mientras que en el aprendizaje en línea el diseño instruccional debe estar explícito en la selección y creación de experiencias que hagan factible el aprendizaje.

\section{Modelos Instruccionales}

- $\quad$ Modelo Assure (1993): Incorpora eventos de instrucción de Robert Gagné para asegurar el uso efectivo de los medios en la instrucción. Tiene sus raíces teóricas en el constructivismo, partiendo de las características concretas del estudiante, sus estilos de aprendizaje y fomentando la participación activa y comprometida del estudiante. Sus siglas indican: Analyse learners, State objectives, Select media and materials, Use media and materials, Requiere learner participation, Evaluate and revise.

Las fases del modelo son:

1. Analizar las características del estudiante. Antes de comenzar, se debe conocer las características de los estudiantes, en relación a:

- Características Generales: nivel de estudios, edad, características sociales, físicas, etc.

- Capacidades específicas de entrada: conocimientos previos, habilidades y actitudes.

- Estilos de Aprendizaje.

2. Establecimiento de objetivos de aprendizaje, determinando los resultados que los estudiantes deben alcanzar al realizar el curso, indicando el grado en que serán conseguidos.

3. Selección de estrategias, tecnologías, medios y materiales. 
- Método Instruccional que se considera más apropiado para lograr los objetivos para esos estudiantes particulares.

- Los medios que serían más adecuados: texto, imágenes, video, audio, y multimedia.

- Los materiales que servirán de apoyo a los estudiantes para el logro de los objetivos.

4. Organizar el escenario de aprendizaje. Desarrollar el curso creando un escenario que propicie el aprendizaje, utilizando los medios y materiales seleccionados anteriormente. Revisión del curso antes de su implementación, especialmente si se utiliza un entorno virtual comprobar el funcionamiento óptimo de los recursos y materiales del curso.

5. Participación de los estudiantes. Fomentar a través de estrategias activas y cooperativas la participación del estudiante.

6. Evaluación y revisión de la implementación y resultados del aprendizaje. La evaluación del propio proceso llevará a la reflexión sobre el mismo y a la implementación de mejoras que redunden en una mayor calidad de la acción formativa.

Modelo de Dick y Carey (1996): Se basa en la idea de que existe una relación predecible y fiable entre un estímulo (materiales didácticos) y la respuesta que se produce en un alumno (el aprendizaje de los materiales).

Las fases del modelo son:

1. Identificar la meta instruccional.

2. Análisis de la instrucción.

3. Análisis de los estudiantes y del contexto.

4. Redacción de objetivos.

5. Desarrollo de Instrumentos de evaluación.

6. Elaboración de la estrategia instruccional.

7. Desarrollo y selección de los materiales de instrucción.

8. Diseño y desarrollo de la evaluación formativa.

9. Diseño y desarrollo de la evaluación sumativa.

10. Revisión de la instrucción

- $\quad$ Modelo de Jonassen (1999): Se enfoca en el diseño de Ambientes de Aprendizaje Constructivistas que enfatiza el papel del aprendiz en la construcción del conocimiento (aprender haciendo).

Las fases del modelo son:

1. Preguntas/casos/problemas/proyectos. El centro de cualquier ambiente de aprendizaje constructivista es la pregunta, caso, problema o proyecto que se convierte en la meta del estudiante a resolver. El problema conduce el aprendizaje, lo cual es la diferencia fundamental entre el ambiente de aprendizaje constructivista y la instrucción objetivista.

- Contexto del problema

- Representación del Problema/simulación

- Espacio de la manipulación del problema

2. Casos relacionados. Ofrecer acceso a un sistema de experiencias relacionadas (casos) como referencia para los estudiantes.

3. Recursos de Información. Los estudiantes necesitan información que les permita construir sus modelos mentales y formular hipótesis que dirijan su actividad en la resolución del problema.

4. Herramientas cognitivas. Al otorgar complejidad, novedad y tareas auténticas, el estudiante necesitará apoyo en su realización. Es importante por tanto, proveerle de herramientas cognitivas que le permitan establecer los andamios o relaciones necesarias en la realización de las mismas.

5. Conversación / herramientas de colaboración. Fomentar y apoyar a comunidades de estudiantes o comunidades que construyen conocimientos a 
través de la comunicación mediada por computadora que apoyan la colaboración y la comunicación.

6. Social / Apoyo del Contexto. Adecuar los factores ambientales y del contexto que afectan a la puesta en práctica del ambiente de aprendizaje constructivista.

Modelo ADDIE (2003): Proceso de diseño Instruccional interactivo, en donde los resultados de la evaluación formativa de cada fase pueden conducir al diseñador instruccional de regreso a cualquiera de las fases previas. El producto final de una fase es el producto de inicio de la siguiente fase. Sus siglas indican: Análisis, Diseño, Desarrollo, Implementación y Evaluación.

Las fases del modelo son:

- Análisis. El paso inicial es analizar el alumnado, el contenido y el entorno cuyo resultado será la descripción de una situación y sus necesidades formativas.

- Diseño. Se desarrolla un programa del curso deteniéndose especialmente en el enfoque pedagógico y en el modo de secuenciar y organizar el contenido.

- Desarrollo. La creación real (producción) de los contenidos y materiales de aprendizaje basados en la fase de diseño.

- Implementación. Ejecución y puesta en práctica de la acción formativa con la participación de los alumnos.

- Evaluación. Esta fase consiste en llevar a cabo la evaluación formativa de cada una de las etapas del proceso ADDIE y la evaluación sumativa a través de pruebas específicas para analizar los resultados de la acción formativa.

De acuerdo a una revisión (Leguizamón, 2011) se puede mencionar que actualmente existen documentadas alrededor de once (11) metodologías (enunciadas en la Tabla numero 1), todas coinciden en establecer como mínimo una etapa de análisis, otra de diseño y/o desarrollo, pruebas y finalmente implementación del producto.

Tabla 1. Metodologías y etapas de desarrollo de software

\begin{tabular}{|l|l|}
\hline Autor y denominación de la metodología & \multicolumn{1}{|c|}{ Etapas } \\
\hline Álvaro Galvis Panqueva. Metodología & 1. Análisis de necesidades educativas \\
para el desarrollo de Materiales & 2. Selección o planeación del desarrollo \\
Educativos Computarizados (MEC). & de MEC \\
& 3. Ciclos para la selección o el desarrollo \\
& de MEC \\
& 4. Diseño de MEC \\
& 5. Entorno para el diseño del MEC \\
& 6. Entorno del diseño \\
& 7. Diseño educativo del MEC \\
& 8. Desarrollo de MEC \\
& 9. Prueba piloto de MEC \\
& 10. Prueba de campo de MEC \\
\hline Ruffini. Aproximación Sistemática y por & 1. Análisis \\
etapas utilizando sistemas multimedia de & 2. Seleccionar el tópico por tratar \\
autor. & 3. Objetivos por cumplir \\
& 4. Definir Proyecto \\
& 5. Diseño de contenidos \\
& 6. Diseño de hipervínculos de navegación \\
\hline La Producción de Soportes Educativos & 7. Evaluación del proyecto multimedia \\
(PROSDOS). & 2. Estudinir tópico, objetivos y contenido \\
& 3. Desarrollo \\
& 4. Estudio del producto \\
& \\
\hline
\end{tabular}




\begin{tabular}{|c|c|}
\hline Autor y denominación de la metodología & Etapas \\
\hline $\begin{array}{l}\text { Ovalle y Padilla. Para el desarrollo de un } \\
\text { Software Educativo Hipermedial (SEH) }\end{array}$ & $\begin{array}{l}\text { 1. Análisis y estudio de factibilidad del } \\
\text { proyecto } \\
\text { 2. Diseño y esquematización pedagógica } \\
\text { de la aplicación. } \\
\text { 3. Desarrollo y programación } \\
\text { 4. Distribución }\end{array}$ \\
\hline Chacón & $\begin{array}{l}\text { 1. Diseño } \\
\text { 2. Producción } \\
\text { 3. Evaluación } \\
\text { 4. Entrega }\end{array}$ \\
\hline $\begin{array}{l}\text { Bertha López Azamar. Gustavo Sergio } \\
\text { Peláez Camarena. María Antonieta Abud } \\
\text { Figueroa. Metodología para el desarrollo } \\
\text { de Software Educativo (DESED) }\end{array}$ & $\begin{array}{l}\text { 1. Determinar la necesidad de un S. E. } \\
\text { 2. Formación del equipo de trabajo } \\
\text { 3. Análisis y delimitación del tema } \\
\text { 4. Definición del usuario } \\
\text { 5. Estructuración del contenido } \\
\text { 6. Elección del tipo de software para } \\
\text { desarrollar } \\
\text { 7. Diseño de interfaces } \\
\text { 8. Definición de las estructuras de } \\
\text { evaluación 9. Elección del ambiente de } \\
\text { desarrollo } \\
\text { 10. Creación de una versión inicial } \\
\text { 11. Prueba de campo } \\
\text { 12. Mercadotecnia } \\
\text { 13. Entrega del producto final }\end{array}$ \\
\hline Pere Marqués. & $\begin{array}{l}\text { 1. La génesis de la idea-semilla } \\
\text { 2. Pre-diseño o diseño funcional } \\
\text { 3. Estudio de viabilidad y marco del } \\
\text { proyecto } 4 \text {. Dossier completo de diseño o } \\
\text { diseño orgánico } \\
\text { 5. Programación y elaboración del } \\
\text { prototipo alfa-test } \\
\text { 6. Redacción de la documentación del } \\
\text { programa } \\
\text { 7. Evaluación interna } \\
\text { 8. Ajustes y elaboración del prototipo } \\
\text { beta-test } \\
\text { 9. Evaluación externa } \\
\text { 10. Ajustes y elaboración de la versión } \\
\text { 1.0 } \\
\text { 11. Publicación y mantenimiento del } \\
\text { producto }\end{array}$ \\
\hline $\begin{array}{l}\text { Ricardo A. Gómez Castro, Álvaro H. } \\
\text { Galvis Panqueva y Olga Mariño Drews. } \\
\text { Metodología ISE-OO }\end{array}$ & $\begin{array}{l}\text { 1. Análisis } \\
\text { 2. Especificación de requerimientos } \\
\text { 3. Diseño } \\
\text { 4. Diseño Educativo } \\
\text { 5. Diseño Comunicacional } \\
\text { 6. Diseño Computacional } \\
\text { 7. Desarrollo } \\
\text { 8. Prueba a lo largo y al final del } \\
\text { desarrollo }\end{array}$ \\
\hline $\begin{array}{l}\text { María Dolores Mendoza Guzmán y } \\
\text { Fernando Gamboa Rodríguez. }\end{array}$ & $\begin{array}{l}\text { 1. Preproducción } \\
\text { 2. Producción }\end{array}$ \\
\hline
\end{tabular}




\begin{tabular}{|c|c|}
\hline Autor y denominación de la metodología & Etapas \\
\hline $\begin{array}{l}\text { Metodología propuesta por el } \\
\text { departamento de multimedia DGSCA } \\
\text { UNAM. }\end{array}$ & 3. Posproducción \\
\hline $\begin{array}{l}\text { Kruchten (1996). Metodología para } \\
\text { desarrollo de software Rational Unified } \\
\text { Process (RUP) }\end{array}$ & $\begin{array}{l}\text { 1. Fase de comienzo o inicio } \\
\text { 2. Fase de elaboración } \\
\text { 3. Fase de construcción } \\
\text { 4. Fase de transición }\end{array}$ \\
\hline $\begin{array}{l}\text { Zulma Cataldi, Fernando Lage, Raúl } \\
\text { Pessacq y Ramón García-Martínez. } \\
\text { Metodología extendida para la creación } \\
\text { de software educativo desde una visión } \\
\text { integradora. }\end{array}$ & $\begin{array}{l}\text { 1. Factibilidad } \\
\text { 2. Definición de requisitos del sistema } \\
\text { 3. Especificación de los requisitos del } \\
\text { prototipo } \\
\text { 4. Diseño del prototipo } \\
\text { 5. Diseño detallado el prototipo } \\
\text { 6. Desarrollo del prototipo } \\
\text { 7. Implementación y prueba del prototipo } \\
\text { 8. Refinamiento iterativo de las } \\
\text { especificaciones del prototipo } \\
\text { 9. Diseño del sistema final } \\
\text { 10. Implementación del sistema final } \\
\text { 11. Operación y mantenimiento } \\
\text { 12. Retiro }\end{array}$ \\
\hline
\end{tabular}




\section{SEGUNDO ANTECEDENTE. MEC}

MEC o Material educativo computarizado (Galvis, 1992) es la denominación otorgada a las diferentes aplicaciones informáticas cuyo objetivo terminal es apoyar el aprendizaje. Se caracterizan porque es el estudiante quien controla el ritmo de aprendizaje, la cantidad de ejercicios, decide cuando abandonar y reiniciar, interactuar reiteradas veces, en fin son muchos los beneficios. Por su parte el docente encuentra en ellos una ayuda significativa, pues en muchos casos en los MECs se registra toda la actividad del estudiante.

\section{TERCER REFERENTE. QUE ES LA COMPUTACION}

Es el estudio sistemático de la factibilidad, estructura, expresión y mecanización de procedimientos metódicos (o algoritmos) que subyacen en la adquisición, representación, procesamiento, almacenamiento, comunicación y acceso a la información si dicha información está codificada en forma de bits en una memoria de computadora o especificada en una estructura de genes y proteínas en una célula biológica (Boston University, 2002).

\section{CUARTO REFERENTE. QUE ES EDUMATICA.}

La informática estudia los procesos de comunicación, tanto de transmisión como de transformación de la información; su obtención, organización y diseminación (Salinas lbáñez, 2008).

La educación es un intento humano racional, intencional de concebirse y perfeccionarse en el ser natural total. Este intento implica apoyarse en el poder de la razón, empleando recursos humanos para continuar el camino del hombre natural hacia el ser cultural. Cada ser humano/ hombre/mujer termina siendo a través de la educación una cultura individual en sí mismo (León Aníbal, 2007).

La Edumatica (FUAC, 1996) se definió como el uso de la informática al servicio de la educación. En Edumatica se estableció la integración de la experiencia (información integral), conocimiento (información disciplinar) y la multimedia (medios como Imagen, Sonido y Texto) en un Sistema Edumático cuyo objetos eran la escena (repositorio de conocimiento, medios y ejercitación), el esquema, set de escenas (integración de escenas que comparten los mismos medios) y navegación (lineal, jerárquica, ir a).

Tabla 2. Categorización de Medios. Fuente Propia

\begin{tabular}{|l|l|l|}
\hline \multicolumn{1}{|c|}{ IMAGEN } & \multicolumn{1}{|c|}{ SONIDO } & \multicolumn{1}{c|}{ TEXTO } \\
\hline $\begin{array}{l}\text { Dibujo: Digitalizados o } \\
\text { hechos por el diseñador }\end{array}$ & Fondo musical & $\begin{array}{l}\text { Descriptivo (describe un } \\
\text { objeto o analiza un } \\
\text { hecho) }\end{array}$ \\
\hline $\begin{array}{l}\text { Animación: Estática o o } \\
\text { secuencia de imágenes y/o } \\
\text { Dinámica desplazamiento } \\
\text { (ejes vertical y horizontal) de } \\
\text { dibujos(s). }\end{array}$ & $\begin{array}{l}\text { Vocal que puede ser } \\
\text { acontecimiento) o } \\
\text { descriptivo (describe un } \\
\text { objeto o analiza un hecho) }\end{array}$ & $\begin{array}{l}\text { Narrativo (narra un } \\
\text { hecho o acontecimiento) }\end{array}$ \\
\hline Video & $\begin{array}{l}\text { Cortina musical al inicio o } \\
\text { cierre de la presentación de } \\
\text { un suceso }\end{array}$ & \\
\hline
\end{tabular}

La Metodología Edumatica tenía las siguientes fases:

1. Análisis de necesidades educativas

- Enfoque del trabajo

- Población objetivo

- Necesidades educativas a apoyar

2. Sistema Edumático - Contenido del sistema

- Medios 
- Marco teórico

- Requerimientos operacionales

- Requerimientos conceptuales

- Requerimientos de hardware y de software

3. Planeación del Proyecto

- Idea o tema a desarrollar

- Cronograma de actividades

4. Propuesta Sistema Edumático

- Factores determinantes para lograr un buen diseño

- Factores que inciden en el diseño de la interfaz

- Estructura del Sistema Edumático

5. Diseño Edumático

- Modelo Esquemático

Escenas / Set de Escenas

Descripción de Medios

Descripción de Escenario

Diseño de Interfaces

Guion

Navegación: Elementos y Representación de Navegación

- Diseño Modular

Módulos

Tabla Visual de Contenido

6. Prototipo y prueba piloto.

Implementación de una escena, probándola con la población objetivo

7. Implementación

Desarrollo del guion utilizando la herramienta de programación seleccionada.

8. Prueba final y ajustes

Verificación de todas las opciones que ofrece el software, haciendo los ajustes que se vayan presentando.

9. Documentación

- Archivos que comprenden el proyecto.

- Manual del usuario

\section{QUINTO REFERENTE. MODELO DE HECKHAUSEN}

Hanz Heckhausen (Heckhausen,1972) expone siete criterios, de lo que debe entenderse como disciplina, "el termino disciplina" se utiliza en el mismo sentido que ciencia", ya que "disciplina" implica la noción de "enseñanza de una ciencia". Los elementos disciplinares son considerados criterios que pueden servir para caracterizar la naturaleza de una disciplina y distinguirla de otras disciplinas:

a. Dominio material: serie de objetos de estudio sobre los cuales trata una disciplina.

b. Dominio de estudio de la disciplina: es el enfoque dado a un objeto de estudio o dominio material.

c. El nivel de integración teórica de la disciplina: es buscar reconstruir la "realidad" de sus dominios de estudio en términos teóricos, con el fin de establecer los conceptos necesarios para comprender, explicar, diagnosticar y pronosticar los acontecimientos y fenómenos de su dominio de estudio.

d. Los métodos de las disciplinas son los procedimientos que permiten captar los fenómenos o resolver los problemas relacionados con ellos.

e. Instrumentos de análisis de las disciplinas pueden ser estrategias lógicas, razonamientos matemáticos, construcción de modelos, etc., que utiliza la disciplina. f. Aplicaciones prácticas de la disciplina sobre todo en el campo de alguna actividad profesional.

g. Contingencias históricas de las disciplinas. Cada disciplina es el fruto de una evolución histórica, y se encuentra, en todo momento, en una fase de transición. 


\section{SEXTO REFERENTE. LA INFORMÁTICA COMO DISCIPLINA CIENTÍFICA Y}

TECNOLÓGICA

La informática como Disciplina Científica y Tecnológica estudia los fenómenos relacionados con los objetos de su dominio conceptual(información y sistemas) y cuenta por un lado, con un conjunto de métodos o procedimientos (modelización y abstracción) que permiten captar y estudiar los fenómenos (tendencias, usabilidad u obsolescencia) relacionados al tratamiento sistémico de la información, y por otro lado, con teorías (teoría de Información y la Comunicación, teoría de las organizaciones) que conceptualizan los objetos de su dominio (Barchine Graciela, 2005).

El dominio Material de la informática (Contreras, 2013) se representa mediante:

- Datos / Información / Conocimiento.

- Sistemas

- Algoritmos

- Sistemas de Información/ Sistemas Basados en Conocimiento

- TI (Tecnologías de la Información).

- Multimedia e Hipertexto 
SEPTIMO REFERENTE. OBJETOS DE CONOCIMIENTO, FORMACIÓN, ESTUDIO El objeto de conocimiento se refiere a la manera concreta como el sujeto piensa al objeto y su definición implica la existencia de protocolos teóricos y o experimentales. El objeto de conocimiento se diferencia por su propio discurso, su problemática concreta y su método de indagación y desarrollo (Arboleda y Lopera, 2002). Por su parte, el objeto de formación está relacionado con la formación profesional, con los procesos educativos para la formación del sujeto e implica el abordaje y dominio de cuerpos de conocimiento teóricos e instrumentales sobre la disciplina o campo del saber (Salazar, 2002). A su vez, los objetos de estudio son procesos que develan potencialidades para descubrir realidades, construir conocimientos, transformar prácticas o recrear saberes y discursos (Jiménez, 2002). Estos objetos determinan el conjunto de saberes o conceptos, actividades, tareas, evidencias esperadas, conceptos a no tratar.

OCTAVO REFERENTE. METODOLOGIA EDUMATICA (Contreras, 2016) Análisis del Sistema Edumático

- Situación Problémica

- Recolección de Información

- Diagnostico

- Formulación del Problema

- Planteamiento del Problema

- Dominio Material y de Aplicación

- Normalización e Identificación del Objeto

- Capa Contenido

- Capa Presentación

- Capa Ayuda

- Navegación

- Estructura de un Componente

Diseño del Sistema Edumático

- Modelo Esquemático

- Descripción del Elemento Terminal (Escena)

Set de Escenas

- Navegación 


\section{Análisis del Sistema Edumático}

En el Análisis Edumático una vez que se haya recolectado la información de la situación problemica, identificado la población objetiva, diagnosticado y formulado el problema Edumático, como también, justificado el sistema Edumático; se plantea el Dominio Material y de Aplicación del tema como resultado de Solución de una Deficiencia o Satisfacción de una Necesidad.

1 Identificación de los objetos de conocimiento, de formación y de estudio del tema

Tabla 3. Tabla de Dominio Material del Tema. Fuente Propia

\begin{tabular}{|c|c|c|}
\hline $\begin{array}{l}\text { Objetos de Conocimiento } \\
\text { (se deben Definir) }\end{array}$ & $\begin{array}{l}\text { Objetos de Formación } \\
\text { (se deben Demostrar) }\end{array}$ & $\begin{array}{l}\text { Objetos de Estudio se deben } \\
\text { saber (Docente) y Aprender } \\
\text { (Estudiante) }\end{array}$ \\
\hline Que se debe Definir & $\begin{array}{l}\text { Como se puede ampliar, } \\
\text { profundizar, } \\
\text { ejemplarizar, jugar }\end{array}$ & $\begin{array}{l}\text { Que se quiere aprender } \\
\text { Que se debe enseñar }\end{array}$ \\
\hline $\begin{array}{l}\text { Recursos como: } \\
\text { Teorías, Teoremas, } \\
\text { leyes, reglas, } \\
\text { metodologías, modelos. }\end{array}$ & $\begin{array}{l}\text { Actividades como: } \\
\text { Aplicaciones, } \\
\text { fenómenos, situaciones, } \\
\text { progresos, posturas, } \\
\text { situaciones, casos de } \\
\text { estudio, lúdica }\end{array}$ & $\begin{array}{l}\text { Es lo que quiere-saber o lo que } \\
\text { debe-saber o lo que debe saber- } \\
\text { hacer un estudiante }\end{array}$ \\
\hline $\begin{array}{l}\text { Los recursos del Objeto } \\
\text { de Conocimiento como } \\
\text { una organización mental } \\
\text { de conceptos(Ámbito } \\
\text { Conceptual) }\end{array}$ & $\begin{array}{l}\text { Los recursos del Objeto } \\
\text { de Conocimiento como } \\
\text { la relación entre } \\
\text { conceptos y actividades. }\end{array}$ & $\begin{array}{l}\text { El objeto de estudio como un } \\
\text { conjunto de conceptos o } \\
\text { representación mental de } \\
\text { conceptos }\end{array}$ \\
\hline \multicolumn{3}{|c|}{$\begin{array}{l}\text { Organizar conceptualmente (Ámbito Conceptual) haciendo uso de uno de estos } \\
\text { recursos: } \\
\text { - Jerarquía de conceptos mediante la relación y/o asociación. } \\
\text { - El Mapa Mental como un diagrama usado para representar las palabras, ideas, } \\
\text { tareas y dibujos u otros conceptos ligados y dispuestos radialmente alrededor de } \\
\text { un objeto. } \\
\text { - El Mapa Conceptual como técnica usada para la representación gráfica de un } \\
\text { Objeto. Un mapa conceptual es una red de Conceptos. } \\
\text { - Y otros recursos }\end{array}$} \\
\hline
\end{tabular}

2 Organización Conceptual del Tema

3 El Tema se presenta inicialmente como un Dominio Material que establece la relación entre el objeto de conocimiento y el objeto de estudio con su ámbito conceptual ante sus medios de presentación.

4 El tema se presenta el tema como el Dominio de Aplicación como la relación entre las actividades y los medios a hacer utilizados.

5 Normalización e Identificación de cada Objeto (Conocimiento, Formación, y de estudio). Se Normaliza cada objeto como Componente (Conocimiento, Formación, y de estudio) y se identifica hacia el interior del Sistema Edumático. Además, para que exista coherencia con lo planteado en el Decimo Referente Arquitectura del Sistema Edumático se establece que la Capa de Contenido será formada por los componentes de Conocimiento, Formación y Estudio.

6 El componente Menú aparece ante la oportunidad de seleccionar o elegir un componente de contenido de una lista de opciones (cada opción es un componente de contenido). Por ejemplo, en un Menú de Continentes existe 
cada continente, por decir, América, Asís, Europa, África, Oceanía como una opción de una lista de opciones.

7 Cada componente de la Capa de Contenido debe tener una estructura como:

Tabla 4. Estructura Componente Capa de Contenido. Fuente Propia

\begin{tabular}{|c|c|c|c|c|}
\hline \multicolumn{5}{|c|}{ CAPA DE PRESENTACION } \\
\hline \multirow[t]{2}{*}{ COMPONENTE } & \multirow{2}{*}{$\begin{array}{l}\text { IDENTIFICACION } \\
\text { COMPONENTE }\end{array}$} & \multicolumn{3}{|c|}{ MEDIOS } \\
\hline & & IMAGEN & SONIDO & TEXTO \\
\hline $\begin{array}{l}\text { Conocimiento } \\
\text { Formación } \\
\text { Estudio } \\
\text { Menú }\end{array}$ & & $\begin{array}{l}\text { Dibujo } \\
\text { Animación } \\
\text { Video }\end{array}$ & $\begin{array}{l}\text { Fondo musical } \\
\text { Vocal Narrativo } \\
\text { Vocal Descriptivo } \\
\text { Cortina musical }\end{array}$ & $\begin{array}{l}\text { Narrativo } \\
\text { Descriptivo }\end{array}$ \\
\hline
\end{tabular}

8 En la Capa de Presentación se puede establecer la tabla de identificación de componentes como:

Tabla 5. Identificación Componentes Capa de Presentación. Fuente Propia

\begin{tabular}{|l|l|l|}
\hline \multicolumn{1}{|c|}{ Componente } & $\begin{array}{l}\text { Identificación como } \\
\text { Componente }\end{array}$ & $\begin{array}{l}\text { Ejemplo de Identificación } \\
\text { de Componente }\end{array}$ \\
\hline Portada & $\begin{array}{l}\text { PO_Objeto (el } \\
\text { nombre de objeto } \\
\text { debe ser titulado) }\end{array}$ & PO_Integrantes \\
\hline Presentación & $\begin{array}{l}\text { PR_Objeto (el } \\
\text { nombre de objeto } \\
\text { debe ser titulado) }\end{array}$ & PR_PresentacionTema \\
\hline Instrucciones & $\begin{array}{l}\text { Pl_Objeto (el nombre } \\
\text { de objeto debe ser } \\
\text { titulado) }\end{array}$ & $\begin{array}{l}\text { PI_Juego } \\
\text { PI_Software }\end{array}$ \\
\hline Agradecimientos & $\begin{array}{l}\text { PA_Objeto (el } \\
\text { nombre de objeto } \\
\text { debe ser titulado) }\end{array}$ & $\begin{array}{l}\text { PA_Familia } \\
\text { PA_Institucion }\end{array}$ \\
\hline Antecedentes & $\begin{array}{l}\text { PN_Objeto (el } \\
\text { nombre de objeto } \\
\text { debe ser titulado) }\end{array}$ & PN_EstudioSocial \\
\hline
\end{tabular}

9 Cada componente de la Capa de Presentación debe tener una estructura como:

Tabla 6. Estructura Componente Capa de Presentación. Fuente Propia

\begin{tabular}{|l|l|l|l|l|}
\hline \multicolumn{5}{|c|}{ CAPA DE PRESENTACION } \\
\hline COMPONENTE & IDENTIFICACION & \multicolumn{3}{c|}{ MEDIOS } \\
\cline { 3 - 5 } & COMPONENTE & IMAGEN & \multicolumn{1}{c|}{ SONIDO } & TEXTO \\
\hline Portada & & Dibujo & Fondo musical & Narrativo \\
Presentación & & Animación & Vocal Narrativo & Descriptivo \\
Instrucciones & & Video & Vocal Descriptivo & \\
Agradecimiento & & & Cortina musical & \\
Antecedentes & & & & \\
\hline
\end{tabular}

10 En la Capa de Ayuda se puede establecer la tabla de identificación de componentes como:

Tabla 7. Capa de Ayuda. Fuente Propia

\begin{tabular}{|l|l|l|}
\hline \multicolumn{1}{|c|}{ Componente } & $\begin{array}{l}\text { Identificación como } \\
\text { Componente }\end{array}$ & $\begin{array}{c}\text { Ejemplo de Identificación } \\
\text { de Componente }\end{array}$ \\
\hline Proyecto & $\begin{array}{l}\text { AP_Objeto (el } \\
\text { nombre de objeto } \\
\text { debe ser titulado) }\end{array}$ & AP_Simbolos \\
\hline Componente & $\begin{array}{l}\text { AC_Objeto (el } \\
\text { nombre de objeto } \\
\text { debe ser titulado) }\end{array}$ & AC_JuegoPar \\
\hline
\end{tabular}



11 Cada componente de la Capa de Ayuda debe tener una estructura como:

Tabla 8. Estructura Componente Capa de Ayuda. Fuente Propia

\begin{tabular}{|c|c|c|c|c|}
\hline \multicolumn{5}{|c|}{ CAPA DE AYUDA } \\
\hline \multirow{2}{*}{ COMPONENTE } & \multirow{2}{*}{$\begin{array}{l}\text { IDENTIFICACION } \\
\text { COMPONENTE }\end{array}$} & \multicolumn{3}{|c|}{ MEDIOS } \\
\hline & & IMAGEN & SONIDO & TEXTO \\
\hline $\begin{array}{l}\text { Proyecto } \\
\text { Componente }\end{array}$ & & $\begin{array}{l}\text { Dibujo } \\
\text { Animación } \\
\text { Video }\end{array}$ & $\begin{array}{l}\text { Fondo musical } \\
\text { Vocal Narrativo } \\
\text { Vocal Descriptivo } \\
\text { Cortina musical }\end{array}$ & $\begin{array}{l}\text { Narrativo } \\
\text { Descriptivo }\end{array}$ \\
\hline
\end{tabular}

12 La navegación se manifiesta como quiere que se aprenda el tema. Entonces, cada componente cumple con ser una secuencia, un menú, una opción de o un llamado de. Por lo tanto, se puede pensar en una navegación lineal o secuencial, navegación por menús, navegación hipermedial donde cada componente es una opción, navegación por hipermedia (un componente es llamado y retorna al componente que llamo)

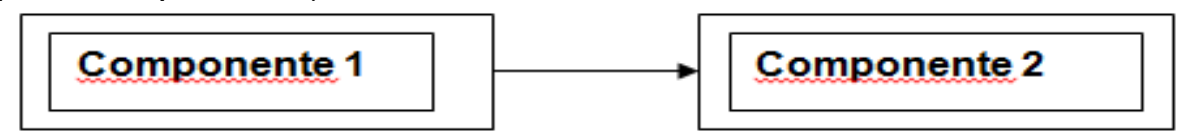

Figura 1. Navegación Lineal. Fuente Propia

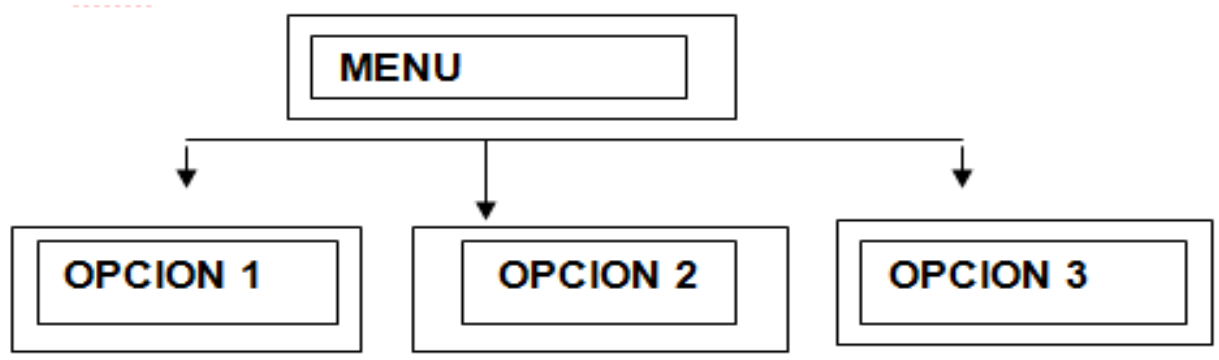

Figura 2. Navegación por Menús. Fuente Propia

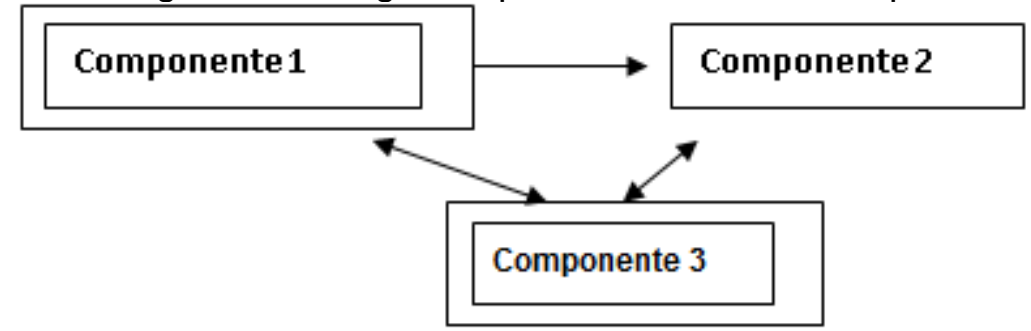

Figura 3. Navegación por Hipermedia. Fuente Propia

13 Se representa cada componente como la integración de una Navegación y un Contenido (Tabla 9). 
Tabla 9. Estructura de un Componente. Fuente Propia

\begin{tabular}{|c|c|c|c|c|}
\hline $\begin{array}{c}\text { COMPONENTE } \\
\text { PREVIO }\end{array}$ & \multicolumn{3}{|c|}{ COMPONENTE } & $\begin{array}{c}\text { COMPONENTE } \\
\text { SIGUIENTE }\end{array}$ \\
\hline $\begin{array}{l}\text { Nombre: } \\
\text { Identificación: } \\
\text { Pertenece a la: } \\
\text { Capa Presentación } \\
\text { - Portada } \\
\text { - Presentación } \\
\text { - Instrucciones } \\
\text { - Agradecimiento } \\
\text {-Antecedentes } \\
\text { Capa Contenido } \\
\text { - Conocimiento } \\
\text { - Formación } \\
\text { - Estudio } \\
\text { - Menú } \\
\text { Capa Ayuda } \\
\text { - Proyecto } \\
\text { - Componente }\end{array}$ & \multicolumn{3}{|c|}{$\begin{array}{l}\text { Nombre: } \\
\text { Identificación: } \\
\text { Pertenece a la: } \\
\text { Capa Presentación } \\
\text { - Portada } \\
\text { - Presentación } \\
\text { - Instrucciones } \\
\text { - Agradecimiento } \\
\text { - Antecedentes } \\
\text { Capa Contenido } \\
\text { - Conocimiento } \\
\text { - Formación } \\
\text { - Estudio } \\
\text { - Menú } \\
\text { Capa Ayuda } \\
\text { - Proyecto } \\
\text { _ Componente }\end{array}$} & $\begin{array}{l}\text { Nombre: } \\
\text { Identificación: } \\
\text { Pertenece a la: } \\
\text { Capa Presentación } \\
\text { - Portada } \\
\text { - Presentación } \\
\text { - Instrucciones } \\
\text { - Agradecimiento } \\
\text { - Antecedentes } \\
\text { Capa Contenido } \\
\text { - Conocimiento } \\
\text { - Formación } \\
\text { - Estudio } \\
\text { - Menú } \\
\text { Capa Ayuda } \\
\text { - Proyecto } \\
\text { - Componente }\end{array}$ \\
\hline \multirow[b]{2}{*}{$\begin{array}{l}\text { Nombre: } \\
\text { Identificación: } \\
\text { Capa Presentación } \\
\text { - Portada } \\
\text { - Presentación } \\
\text { - Instrucciones } \\
\text { - Agradecimiento } \\
\text { - Antecedentes } \\
\text { Capa Contenido } \\
\text { Conocimiento } \\
\text { - Formación } \\
\text { - Estudio } \\
\text { - Menú } \\
\text { Capa Ayuda } \\
\text { - Proyecto } \\
\text { - Componente }\end{array}$} & \multicolumn{3}{|c|}{ MEDIOS } & Nombre: \\
\hline & \begin{tabular}{l}
\multicolumn{1}{l}{ IMAGEN } \\
Dibujo \\
Animación \\
Video
\end{tabular} & $\begin{array}{l}\text { SONIDO } \\
\text { Fondo } \\
\text { musical } \\
\text { Vocal } \\
\text { Narrativo } \\
\text { Vocal } \\
\text { Descriptivo } \\
\text { Cortina } \\
\text { musical }\end{array}$ & $\begin{array}{l}\text { TEXTO } \\
\text { Narrativo } \\
\text { Descriptivo }\end{array}$ & $\begin{array}{l}\text { Identificación: } \\
\text { Capa Presentación } \\
\text { - Portada } \\
\text { - Presentación } \\
\text { - Instrucciones } \\
\text { - Agradecimiento } \\
\text { - Antecedentes } \\
\text { Capa Contenido } \\
\text { - Conocimiento } \\
\text { - Formación } \\
\text { - Estudio } \\
\text { - Menú } \\
\text { Capa Ayuda } \\
\text { - Proyecto } \\
\text { - Componente }\end{array}$ \\
\hline
\end{tabular}

14 En la Capa de Ayuda se puede establecer la tabla de identificación de componentes como:

Tabla 10. Capa de Ayuda. Fuente Propia

\begin{tabular}{|l|l|l|}
\hline \multicolumn{1}{|c|}{ Componente } & $\begin{array}{l}\text { Identificación como } \\
\text { Componente }\end{array}$ & $\begin{array}{l}\text { Ejemplo de Identificación } \\
\text { de Componente }\end{array}$ \\
\hline Proyecto & $\begin{array}{l}\text { AP_Objeto (el } \\
\text { nombre de objeto } \\
\text { debe ser titulado) }\end{array}$ & AP_Simbolos \\
\hline Componente & $\begin{array}{l}\text { AC_Objeto (el } \\
\text { nombre de objeto } \\
\text { debe ser titulado) }\end{array}$ & AC_JuegoPar \\
\hline
\end{tabular}


15 Cada componente de la Capa de Ayuda debe tener una estructura como:

Tabla 11. Estructura Componente Capa de Ayuda. Fuente Propia

\begin{tabular}{|c|c|c|c|c|}
\hline \multicolumn{5}{|c|}{ CAPA DE AYUDA } \\
\hline \multirow{2}{*}{ COMPONENTE } & \multirow{2}{*}{$\begin{array}{l}\text { IDENTIFICACION } \\
\text { COMPONENTE }\end{array}$} & \multicolumn{3}{|c|}{ MEDIOS } \\
\hline & & IMAGEN & SONIDO & TEXTO \\
\hline $\begin{array}{l}\text { Proyecto } \\
\text { Componente }\end{array}$ & & $\begin{array}{l}\text { Dibujo } \\
\text { Animación } \\
\text { Video }\end{array}$ & $\begin{array}{l}\text { Fondo musical } \\
\text { Vocal Narrativo } \\
\text { Vocal Descriptivo } \\
\text { Cortina musical }\end{array}$ & $\begin{array}{l}\text { Narrativo } \\
\text { Descriptivo }\end{array}$ \\
\hline
\end{tabular}

\section{Diseño Sistema Edumático}

1. Modelo Esquemático

Esquema es la representación gráfica del Proyecto/Software Edumático.

Se aplica el concepto de:

Globalización. El proyecto es visto como un todo

Especialización. Se divide el proyecto de acuerdo al contenido; cada división es una Especialización del Elemento Global.

Entonces se puede decir en lenguaje natural:

- Forma1. La División es Parte de un Proyecto

- Forma2. Proyecto está compuesto de una división

A su vez esta división pasa a ser Global. Esta división a su vez se divide sucesivamente hasta llegar a un elemento terminal (Escena) o un Set de Escenas Entonces cada escena representa un Componente.

Entonces un Set de Escenas es la integración de varios Componentes en una sola escena (no se debe confundir con componente Menú).

Cada escena se identifica ídem que su componente asociado.

2. Descripción de una Escena

- Medios

- Escenario

- Áreas

3. Set de Escenas

4. Guion

5. Navegación 


\section{REFERENCIAS BIBLIOGRAFICAS}

Arboleda, O. y Lopera, D. (2002). Objetos de conocimiento. Textos y Argumentos, 4, Medellín. Fundación Universitaria Luis Amigó

Benitez, M.G. (2010). El modelo de diseño instruccional Assure aplicado a la educación a distancia. Tlatemoani, Revista Académica de Investigación, №1. Disponible en http://www.eumed.net/rev/tlatemoani/01/pdf/63-77_mgbl.pdf

Berger, C. \& Kam, R. (1996). Definitions of Instructional Design. Adapted from "Training and Instructional Design". Applied Research Laboratory, Penn State University. Disponible: http://www.umich.edu/ ed626/define.html

Barchine, G (2005). Una disciplina Bio-Psico-Socio-Tecno-cultural, consultada en febrero 10 del 2009, disponible en http://www.inf.udec.cl/ revista/ediciones/edicion12/articulo\%2012-3.pdf

Blumschein, P.; Fischer, M. (2007). E-learning en la formación profesional: diseño didáctico de acciones de e-learning. Montevideo: Cinterfor/OIT. Disponible en http://www.cinterfor.org.uy/public/spanish/region/ampro/cinterfor/publ/inwe $\mathrm{nt} /$

Boston University, Department of Computer Science (2002). What is Computer Science. Fuente: http://www.cs.bu.edu/AboutCS/WhatlsCS.pdf

Bruner, J. S. (1969). Hacia una teoría de la instrucción. México: UTEHA.

Coll, C. Mauri, T. y Onrubia, J. (2008). Los entornos virtuales de aprendizaje basados en el análisis de casos y la resolución de problemas. En Psicología de la educación virtual, editado por C. Coll y C. Monereo. España: Morata.

Contreras, Mario (2013). Proyecto Educativo del Programa (PEP) en Ingeniería en Informática Universidad Santo Tomás. Disponible en https://docs.google.com/document/d/1HWMsg9K36rCWNvmUbp0lqxMA4eU0JnyZeJC ECHfMr-M/pub

Contreras, Mario (2016). Libro Diseño Edumático. Registro DNDA (Dirección Nacional de Derechos de Autor en Colombia) 10-600-493.

Díaz Barriga, F. (2006). Principios de diseño instruccional de entornos de aprendizaje apoyados en TIC: un marco de referencia sociocultural y situado. Tecnología y Comunicación Educativa, 41. Disponible en http://investigacion.ilce.edu.mx/tyce/41/art1.pdf

Dorrego, E. (1999). Flexibilidad en el diseño instruccional y nuevas tecnologías de la información y comunicación. Compilación con fines instruccionales. Disponible en http://especializacion.una.edu.ve/teoriasaprendizaje/paginas/Lecturas/Unidad 3/dorregoflexi.pdf 
FUAC, Fundación Universidad Autonoma de Colombia (1996), Diseño Edumático. Postgrado Edumatica. Autores: Mario Contreras, Jairo Cortes, Jorge Salazar, Hector Arias, Luis Burbano.

Galvis Panqueva, A. (1992). Ingeniería de Software Educativo. Bogotá: Ediciones Uniandes

Guardia, L. (2000). El Diseño formativo: Un enfoque del diseño pedagógico de los materiales didácticos en soporte digital. En J. M. Duart y A. Sangrà (Compl.): Aprender en la virtualidad. Barcelona: GEDISA, pp. 171 a 187.

Jiménez, L. (2002). Objetos de estudio. Textos y Argumentos. Medellín, Fundación Universitaria Luis Amigó.

Jonassen, D.H. (1994). Thinking Technology. Toward a Constructivist Design Model. Educational Technology. USA.

Leguizamon, M (2011). Diseño y desarrollo de materiales educativos computarizados (mec's): una posibilidad para integrar la informática con las demás áreas del currículo. Fuente: http://www.colombiaaprende.edu.co/html/mediateca/1607/articles106492_archivo.pdf

Leon, Anibal(2007).Qué es la educación, Educere, vol. 11, núm. 39, octubre-diciembre, 2007, pp. 595-604.

Mergel, B. (1998). Diseño instrucccional y teoría de aprendizaje. Occasional Papers in Educational Technology. Disponible en http://www.usask.ca/education/coursework/802papers/mergel/espanol.pdf

Nieto, M. (2010). Diseño instrucional: elementos básicos del diseño instruccional. Publicación en línea. Disponible en http://es.scribd.com/doc/33372131/DISENO-INSTRUCCIONAL-TEORIAS-YMODELOS

Reigeluth, C. M. (1983). Meaningfulness and Instruction: Relating What Is Being Learned to What a Student Knows. Instructional Science, v12 n3 p197-218 Oct 1983, 12(3).

Salazar, N. (2002). Objetos de formación. Textos y Argumentos,4, Medellín, Fundación Universitaria Luis Amigó

Salina Ibañez, Jesús. Libro Innovación Educativo y uso de las TIC. Universidad Internacional de Andalucía, 2008. ISBN: 978-84-7993-055-4

Winn, W. (1991). The assumptions of Constructivism and Instructional Design. Educational Technology. USA.

Williams, P., Schrum, L., Sangra, A. y Guardia, L. Modelos de diseño instruccional. Material didáctico web de la UOC. Publicación en línea. Disponible en http://aulavirtualkamn.wikispaces.com/file/view/2.+MODELOS+DE+DISE\%C3\% 910+INSTRUCCIONAL.pdf 